\title{
Improved Estimation of Sensitive Mean Using Hybrid of Partial and Optional Scrambling in the Presence of Non- Sensitive Auxiliary Information
}

\author{
Zawar Hussain $^{1 *}$ and Waqas Arshad ${ }^{2 * *}$ \\ ${ }^{1}$ Department of Social and Allied Sciences, Cholistan University of Veterinary and \\ Animal Sciences, Bahawalpur 63100, Pakistan. \\ ${ }^{2}$ Department of Statistics, Quaid-i-Azam University, Islamabad 44000, Pakistan. \\ Emails: *zhlangah@yahoo.com, ${ }^{* *}$ waqas_qau@yahoo.com
}

Received: $3^{\text {rd }}$ Oct. 2018/ Revised: $15^{\text {th }}$ Dec. $2018 /$ Published: $30^{\text {th }}$ Dec. 2018 CIAppStat-SL2018

\begin{abstract}
This article is about studying ratio, product and regression methods for estimating sensitive mean using a two-stage optional randomized response model by Gupta et al. (2010) and information on non-sensitive auxiliary variable. In particular, the additive randomized response model is used to further enhance the efficiency of the ratio, product and regression estimators (Gupta et al., 2010). We compare our proposed auxiliary information based two-stage optional randomized response estimator with recently proposed auxiliary information-based estimators. Through algebraic comparisons, it is shown that the proposed ratio, product and regression estimators are better than the corresponding estimators proposed in some recent studies. The results are also supported by a numerical study.
\end{abstract}

Key words: Two-stage optional randomized response model, mean squared error, ratio estimator, product estimator and regression estimator.

\section{Introduction}

Since long, the sample survey has been appreciated as a go to method for the procurement of data from several areas of human interest. For instance, in political science, Cronin Jr. et al. (2000) recognized the convenience, handiness, functionality and practicality of human surveys and mentioned it as a mostly used method for understanding politics. To have more insight about this research area 
One may refer to compelling accounts of Mahoney and Rueschemeyer (2003) and McNabb (2015). Other than this, a rich number of applications of surveys can be observed in different fields of interest like demography (Kellogg (2014) and Abel, et al. (2016)), policy making (Eurostate - household income and expenditure survey and U.S. Bureau of Justice Statistics surveys), epidemiology (Dawidowska et al. (2012) and Ojikutu and Bolanle (2018)), psychology (Karelaia and Hogarth (2008) and Robinson and Irwin (2019)) etc.

While studying stigmatizing behaviors, avoiding the social desirability bias (SDB) is the main challenge for survey practitioners. The main reason for observing the SDB is the natural tendency of respondents to show possession of socially approved (accepted) traits. For a more detailed explanation of this issue, one may refer to Groves et al. (2009), Kelly et al. (2013) and Schill and Kirk (2017). Some of the survey practitioners have also noted troublesome points about the negative effect of SDB on the correctness of results obtained through surveys. Some of them are noted here as Heijden et al. (2000) observed that $75 \%$ of the respondents who had committed welfare or unemployment benefit fraud denied having done so in faceto-face interviews; Lee and Sargeant (2011) noted that $65 \%$ of the respondents over-reported their donations; Stecklov et al. (2015), observed that high reported use of sterilization is correlated with propensity of respondents to present themselves in a positive way in front of interviewers.

Amongst many, the most effective approach to deal with the problem of SDB is the randomized response techniques (RRT), initially, presented by Warner (1965). Over the time, it was further developed and applied by Greenberg et al. (1969), Horvitz et al. (1967), Himmelfarb and Edgell (1980), Chaudhuri and Mukerjee (1985), Kuk (1990), Mangat and Singh (1990), Mangat (1994), Tracy and Mangat (1996), Mahmood et al. (1998), Bhargava and Singh (2000), Gupta et al. (2002), Singh et al. (2003), Bar-Lev et al. (2004), Ryu et al. (2006), Gupta et al. (2006), Gjestvang and Singh (2007), Huang (2008, 2010), and many others. Recently, Kuokkanen et al. (2017) praised RRTs as "more sophisticated methods for SDB reduction". Further, Lensvelt-Mulders et al. (2005) in their extensive validity examination of 38 RRTs noted that "the more sensitive the topic under investigation, the higher the validity of $R R$ results". For further details, comprehending the effectiveness of RRTs, in study of sensitive attributes, one may also refer the works done by Blume et al. (2007), Krumpal (2012), Cruyff et al. (2016) and Shah, et al. (2020a,b).

Over the years, it has been established that the use of non-sensitive auxiliary information is very helpful in increasing the efficiency of the estimates. In the conventional randomization method, scrambled information on only the sensitive study variable is collected. More recently, auxiliary information in sensitive surveys 
has been used quiet fruitfully by many authors like ZaiZai (2005-2006) in ratio type estimation of population proportion based on Warner (1965) model; Kadilar and Cingi (2006), Diana and Perri (2007), Turgut and Cingi (2008), Koyuncu and Kadilar (2009) and Shabbir and Gupta (2010) in ratio, product and regression estimation of mean of a sensitive variable. Motivated by the successful applications of auxiliary information in sensitive surveys, we intend to make use of auxiliary information along with a two-stage optional randomized response model of Gupta et al. (2010) to suggest ratio, product and regression estimators. Specifically, the intended ratio, product and regression estimators are based on one non-sensitive auxiliary variable. In order to have a comparative study, we plan to derive the expressions for bias and mean squared error (MSE) of proposed ratio, product and regression estimators using two-stage optional randomized responses and compare them with different corresponding ratio, product and regression estimators.

In rest of the article, we briefly present, in section 2 , some background information on ratio, product and regression estimators based on non-sensitive auxiliary information and scrambled information, obtained through different RRTs, on sensitive variable. In section 3, we discuss a two-stage optional randomized response model to be used later. In section 4, firstly, we propose ratio, product and regression estimators and then find the expressions for bias and MSE of the proposed estimators. In section 5, we compare, numerically and theoretically, the proposed two-stage optional randomized response-based estimators with already existing recent estimators and establish the unconditional superiority of the proposed estimators. Concluding remarks are given in the section 6 .

\section{Some recent RRTs and associated estimators}

In this section, we discuss some non-sensitive auxiliary information based on randomized response estimators suggested by Sousa et al. (2010), Gupta et al. 2012) and Gupta et al. (2014).

\subsection{Additive scrambling model (Sousa et al. , 2010)}

Let $X$ be a sensitive study variable, $Y$ be a non-sensitive auxiliary variable and $\mathrm{S}$ be a scrambling variable. As study variable is sensitive in nature, it cannot be observed directly. Sousa et al. (2010) used an additive scrambled response model where each respondent, selected in a random sample of size $n$ drawn from a finite population, is asked to report a scrambled response, say $Z$ given by $Z=X+S$ and true response on the auxiliary variable $Y$. 
Let $\bar{x}=\frac{\sum_{i=1}^{n} x_{i}}{n}, \bar{y}=\frac{\sum_{i=1}^{n} y_{i}}{n}$ and $\bar{z}=\frac{\sum_{i=1}^{n} z_{i}}{n}$ be the sample means.

Also, let $E(X)=\mu_{X}, E(Y)=\mu_{Y}, E(Z)=\mu_{Z} \quad$ and $\quad E(S)=\mu_{S}=0 \quad$ be the population means.

The mean $\mu_{Y}$ is assumed to be known and $E(X)=E(Z)$. The usual mean per unit unbiased estimator of $\mu_{X}$, suggested by Sousa et al. (2010), is given by

$$
\widehat{\mu}_{X S}=\bar{z}
$$

Its variance is given by

$$
\operatorname{Var}\left(\widehat{\mu}_{X S}\right)=\operatorname{MSE}\left(\widehat{\mu}_{X S}\right)=\left(\frac{1-f}{n}\right)\left(\sigma_{X}^{2}+\sigma_{S}^{2}\right),
$$

where $f=\frac{n}{N}, \sigma_{X}^{2}=\frac{1}{N-1} \sum_{i=1}^{N}\left(x_{i}-\mu_{X}\right)^{2}$ and $\sigma_{S}^{2}=\frac{1}{N-1} \sum_{i=1}^{N}\left(s_{i}-\mu_{S}\right)^{2}$. Sousa et al. (2010) also suggested a ratio estimator of $\mu_{X}$ which is given by

$$
\hat{\mu}_{R S}=\left(\frac{\bar{z}}{\bar{y}}\right) \mu_{Y}
$$

The bias and MSE of the $\hat{\mu}_{R S}$ are given by

$$
\begin{aligned}
& \operatorname{Bias}\left(\hat{\mu}_{R S}\right) \cong\left(\frac{1-f}{n}\right) \mu_{X}\left(C_{Y}^{2}-\rho_{Y Z} C_{Z} C_{Y}\right) \\
& \operatorname{MSE}\left(\hat{\mu}_{R P}\right) \cong\left(\frac{1-f}{n}\right) \mu_{X}^{2}\left(C_{Z}^{2}+C_{Y}^{2}-2 \rho_{Y Z} C_{Z} C_{Y}\right),
\end{aligned}
$$

Where $\rho_{Y Z}=\frac{\rho_{X Y}}{\sqrt{1+\frac{\sigma_{S}^{2}}{\sigma_{X}^{2}}}}$ the correlation coefficient between $\mathrm{Y}$ and $\mathrm{Z}$. $C_{Z}^{2}=C_{X}^{2}+\frac{\sigma_{S}^{2}}{\mu_{X}^{2}}$ is the squared coefficient of variation of $Z$. 
Using the same additive scrambled response model of Sousa et al. (2010), Gupta et al. (2012) suggested regression type estimator of the mean $\mu_{X}$, given by

$$
\hat{\mu}_{\operatorname{Re} g G}=\bar{z}+\hat{\beta}_{Z Y}\left(\mu_{Y}-\bar{y}\right)
$$

where $\hat{\beta}_{Z Y}$ is the sample regression coefficient between $Z$ and $Y$.The expressions for the bias and MSE of the regression estimator are given as

$$
\operatorname{Bias}\left(\hat{\mu}_{\mathrm{Re} g}\right) \cong-\beta_{Z Y}\left(\frac{1-f}{n}\right)\left\{\frac{\mu_{12}}{\mu_{11}}-\frac{\mu_{03}}{\mu_{02}}\right\},
$$

Where $\beta_{Z Y}=\beta_{X Y}, f=\frac{n}{N}$ and $\mu_{r s}=\frac{1}{N-1} \sum_{i=1}^{N}\left(z_{i}-\bar{Z}\right)^{r}\left(y_{i}-\bar{Y}\right)^{s}$.

$$
\operatorname{MSE}\left(\widehat{\mu}_{\mathrm{Re} g G}\right) \cong\left(\frac{1-f}{n}\right) \mu_{Z}^{2} C_{Z}^{2}\left\{1-\rho_{Y Z}^{2}\right\},
$$

\subsection{Gupta et al. (2014) optional additive scrambling model}

Gupta et al. (2014) used an optional randomized response to estimate the mean of a sensitive variable. The $i^{\text {th }}$ respondent in a sample of size $n$ is requested to provide an additive scrambled response $Z^{\prime}=X+S V$, where $V$ is a Bernoulli random variable taking value $1(0)$, if the study variable is sensitive (insensitive) to a particular respondent. The respondents are also requested to give true response on the auxiliary variable. The usual unbiased estimator of the population mean is given by

$$
\widehat{\mu}_{X W}=\vec{z} .
$$

The MSE of the estimator $\hat{\mu}_{X W}$ is given by

$$
\operatorname{Var}\left(\widehat{\mu}_{X W}\right)=\operatorname{MSE}\left(\widehat{\mu}_{X W}\right)=\left(\frac{1-f}{n}\right)\left(\sigma_{X}^{2}+W \sigma_{S}^{2}\right),
$$

where $f, \sigma_{X}^{2}$ and $\sigma_{S}^{2}$ are defined as above. 
The ratio estimator, suggested by Gupta et al. (2014), is given by

$$
\hat{\mu}_{R W G}=\left(\frac{\vec{z}}{\bar{y}}\right) \mu_{Y} .
$$

The bias and MSE of $\hat{\mu}_{R W G}$ are given by

$$
\begin{aligned}
& \operatorname{Bias}\left(\hat{\mu}_{R W G}\right) \cong\left(\frac{1-f}{n}\right) \mu_{Z^{\prime}}\left(C_{Y}^{2}-\rho_{Y Z^{\prime}} C_{Z^{\prime}} C_{Y}\right) \\
& \operatorname{MSE}\left(\hat{\mu}_{R W G}\right) \cong\left(\frac{1-f}{n}\right) \mu_{Z^{\prime}}^{2}\left(C_{Z^{\prime}}^{2}+C_{Y}^{2}-2 \rho_{Y Z^{\prime}} C_{Z^{\prime}} C_{Y}\right),
\end{aligned}
$$

Where $\quad \rho_{Y Z^{\prime}}=\frac{\rho_{X Y}}{\sqrt{1+W \frac{\sigma_{S}^{2}}{\sigma_{X}^{2}}}}, \quad$ and $\quad C_{Z^{\prime}}^{2}=C_{X}^{2}+W \frac{\sigma_{S}^{2}}{\mu_{X}^{2}}$.

The regression estimator, suggested by Gupta et al. (2014), is given by

$$
\hat{\mu}_{\operatorname{Re} g W G}=\vec{z}^{\prime}+\hat{\beta}_{Z^{\prime} Y}\left(\mu_{Y}-\bar{y}\right),
$$

where $\hat{\beta}_{Z^{\prime} Y}$ is the sample regression coefficient between $Z^{\prime}$ and $Y$.

The bias and MSE of the regression estimator $\hat{\mu}_{\operatorname{Re} g W G}$ are given by

$$
\begin{aligned}
& \operatorname{Bias}\left(\hat{\mu}_{\mathrm{Re} g W G}\right) \cong-\beta_{Z^{\prime} Y}\left(\frac{1-f}{n}\right)\left\{\frac{\mu_{12}}{\mu_{11}}-\frac{\mu_{03}}{\mu_{02}}\right\}, \\
& \operatorname{MSE}\left(\hat{\mu}_{\mathrm{Re} g W G}\right) \cong\left(\frac{1-f}{n}\right) \mu_{Z^{\prime}}^{2} C_{Z^{\prime}}^{2}\left\{1-\rho_{Y^{\prime}}^{2}\right\},
\end{aligned}
$$

where $\rho_{Y Z^{\prime}}$ and $C_{Z^{\prime}}^{2}$ are defined as above. Gupta et al. (2014) compared their regression estimator of mean with the regression estimator suggested by Gupta et al. (2012) and showed that their estimator performed better when $\rho_{X Y}^{2}>0$. 
Under the same optional RRT, if we apply the product estimation of mean, then the product estimator may be suggested as

$$
\hat{\mu}_{P W G}=\left(\frac{\bar{y}}{\mu_{Y}}\right) \vec{z}^{\prime}
$$

with bias and MSE given by

$$
\begin{aligned}
& \operatorname{Bias}\left(\hat{\mu}_{P W G}\right) \cong\left(\frac{1-f}{n}\right) \mu_{Z^{\prime}} \rho_{Y Z^{\prime}} C_{Z^{\prime}} C_{Y}, \\
& \operatorname{MSE}\left(\hat{\mu}_{P W G}\right) \cong\left(\frac{1-f}{n}\right) \mu_{Z^{\prime}}^{2}\left(C_{Z^{\prime}}^{2}+C_{Y}^{2}+2 \rho_{Y Z^{\prime}} C_{Z^{\prime}} C_{Y}\right) .
\end{aligned}
$$

\section{Two Stage Optional Randomized Response Model}

Gupta et al. (2010) proposed a two-stage optional RRT. In this technique, a random sample of size $n$ is drawn from population of size $N$. As discussed earlier, the study variable $X$ and the auxiliary variable $Y$ are correlated and the correlation between $X$ and $Y$ is denoted by $\rho_{X Y}$. Also, the scrambling variable $S$ is assumed to have mean $\mu_{S}=0$ and known variance $\sigma_{S}^{2}$. The whole procedure was performed in two stages. At $1^{\text {st }}$ stage, a randomly selected fixed proportion $(T)$ of the selected respondent give the true response $X$ and the remaining proportion $(1-T)$ of the Respondents is directed to go to stage 2. At stage 2, respondents were given an option to additively scramble their response if they feel study variable sensitive, otherwise, they were asked to report the true response on the sensitive variable. The distribution of the reported response, say $Z^{*}$, is given by:

$$
Z^{*}=\left\{\begin{array}{cl}
X & \text { with probability } T+(1-T)(1-W) \\
S+X & \text { with probability }(1-T) W,
\end{array}\right.
$$

where $W$ is named as the sensitivity level. The expected response may be written as

$$
E\left(Z^{*}\right)=\mu_{X}+(1-T) W \mu_{S}
$$

Now, after replacing the value of $\mu_{S}=0$, we get

$$
E\left(Z^{*}\right)=\mu_{X}
$$


The usual unbiased estimator of the $\mu_{X}$, suggested by Gupta et al. (2010), is given by

$$
\hat{\mu}_{X P}=\bar{z}^{*} \text {, }
$$

Where $\quad \bar{z}^{*}=\frac{\sum_{i=1}^{n} z_{i}^{*}}{n}$ and

its variance is given by

$$
\operatorname{Var}\left(\hat{\mu}_{X P}\right)=\operatorname{MSE}\left(\hat{\mu}_{X P}\right)=\left(\frac{1-f}{n}\right)\left(\sigma_{X}^{2}+W(1-T) \sigma_{S}^{2}\right),
$$

\section{Proposed Ratio, Product and Regression estimators}

We now suggest the ratio, product and regression estimators under the Gupta et al. (2010) RRT. The reason behind taking Gupta et al. (2010) RRT is the advantage of distributing the total probability of reporting on sensitive variable into two stages which, in turn, results in more cooperation from the respondents in terms of truthful reporting.

\subsection{Ratio Estimator}

Now, we propose the ratio estimator of the population mean which is based on the true information on one non-sensitive auxiliary variable $Y$. The proposed ratio estimator of mean is given by

$$
\hat{\mu}_{R P}=\left(\frac{\bar{z}^{*}}{\bar{y}}\right) \mu_{Y}
$$

The estimator $\quad \hat{\mu}_{R P} \quad$ can also be written as

$$
\hat{\mu}_{R P}=\mu_{z^{*}}\left(1+e_{Z^{*}}\right)\left(1+e_{Y}\right)^{-1},
$$

Where $e_{Z^{*}}=\frac{\bar{z}^{*}-\mu_{Z^{*}}}{\mu_{Z^{*}}}$ and $e_{Z}=\frac{\bar{y}-\mu_{Y}}{\mu_{Y}}$.

Using Taylor's series expansion and first order approximation, we have

$$
\hat{\mu}_{R P}-\mu_{Z} \cong \mu_{Z^{*}}\left(e_{Z^{*}}-e_{Y}-e_{Y} e_{Z^{*}}+e_{Y}^{2}\right),
$$


The Bias and MSE of $\hat{\mu}_{R p}$ are given by

$$
\begin{aligned}
& \operatorname{Bias}\left(\hat{\mu}_{R P}\right) \cong\left(\frac{1-f}{n}\right) \mu_{Z^{*}}\left(C_{Y}^{2}-\rho_{Y Z^{*}} C_{Z^{*}} C_{Y}\right) \\
& \operatorname{MSE}\left(\hat{\mu}_{R P}\right) \cong\left(\frac{1-f}{n}\right) \mu_{Z^{*}}^{2}\left(C_{Z^{*}}^{2}+C_{Y}^{2}-2 \rho_{Y Z^{*}} C_{Z^{*}} C_{Y}\right),
\end{aligned}
$$

Where $\quad E\left(e_{Z^{*}}\right)=E\left(e_{Y}\right)=0, \quad E\left(e_{Z^{*}}^{2}\right)=\left(\frac{1-f}{n}\right) C_{Z^{*}}^{2}, \quad E\left(e_{Y}^{2}\right)=\left(\frac{1-f}{n}\right) C_{Y}^{2}$ $E\left(e_{Z^{*}} e_{Y}\right)=\left(\frac{1-f}{n}\right) \rho_{Y Z^{*}} C_{Z^{*}} C_{Y}$,

$$
\rho_{Y Z^{*}}=\frac{\rho_{X Y}}{\sqrt{1+W(1-T) \frac{\sigma_{S}^{2}}{\sigma_{X}^{2}}}}
$$

and

$C_{Z^{*}}^{2}=C_{X}^{2}+W(1-T) \frac{\sigma_{S}^{2}}{\mu_{X}^{2}}$

It is to be noted that the covariance between $X$ and $Y$ is equal to the covariance between $Z^{*}$ and $Y$, that is, $\sigma_{X Y}=\sigma_{Z^{*} Y}$

Now, substituting the value of $\rho_{Y Z^{*}}$ and $C_{Z^{*}}^{2}$ in the expressions of bias and MSE, we get:

$$
\begin{aligned}
& \operatorname{Bias}\left(\hat{\mu}_{R P}\right) \cong\left(\frac{1-f}{n}\right) \mu_{X}\left(C_{Y}^{2}-\frac{\rho_{X Y}}{\sqrt{1+W(1-T) \frac{\sigma_{S}^{2}}{\sigma_{X}^{2}}}} \sqrt{\left(C_{X}^{2}+W(1-T) \frac{\sigma_{S}^{2}}{\mu_{X}^{2}}\right)} C_{Y}\right) \\
& \operatorname{MSE}\left(\hat{\mu}_{R P}\right)=\left(\frac{1-f}{n}\right) \mu_{X}^{2}\left(C_{X}^{2}+W(1-T) \frac{\sigma_{S}^{2}}{\mu_{X}^{2}}+C_{Y}^{2}-2 \frac{\rho_{X Y}}{\sqrt{\left(1+W(1-T) \frac{\sigma_{S}^{2}}{\sigma_{X}^{2}}\right)}} \sqrt{\left(C_{X}^{2}+W(1-T) \frac{\sigma_{S}^{2}}{\mu_{X}^{2}}\right)} C_{Y}\right)
\end{aligned}
$$


It is interesting to observe that for $T=0$ and $W=1, \operatorname{MSE}\left(\hat{\mu}_{R P}\right)$ coincides with the MSE of ratio estimator by Sousa et al. (2010) and for $T=0$, the MSE of our proposed ratio estimator is exactly the same as the ratio estimator proposed by Gupta et al. (2014).

\subsection{Product Estimator}

Based on the data obtained by Gupta et al. (2010) optional RRT, the proposed product estimator is given as:

$$
\hat{\mu}_{P P}=\frac{\bar{y} \cdot \bar{z}^{*}}{\mu_{Y}},
$$

Following the steps discussed in section 4.1, the bias and MSE of the proposed product estimator $\hat{\mu}_{P P}$ are given by

$$
\begin{aligned}
& \operatorname{Bias}\left(\hat{\mu}_{P P}\right)=\left(\frac{1-f}{n}\right) \mu_{Z^{*}} \rho_{Z^{*}} C_{Z^{*}} C_{Y}, \\
& \operatorname{MSE}\left(\hat{\mu}_{P P}\right)=\left(\frac{1-f}{n}\right) \mu_{Z^{*}}^{2}\left(C_{Z^{*}}^{2}+C_{Y}^{2}+2 \rho_{Y Z^{*}} C_{Z^{*}} C_{Y}\right),
\end{aligned}
$$

Now, replacing the value of $C_{Z^{*}}^{2}$ and $\rho_{Y Z^{*}}$ in above expressions, we get the expression of Bias and MSE of $\hat{\mu}_{P P}$ as follows:

$$
\begin{gathered}
\operatorname{Bias}\left(\hat{\mu}_{P P}\right) \cong\left(\frac{1-f}{n}\right) \mu_{X} \frac{\rho_{X Y}}{\sqrt{1+W(1-T) \frac{\sigma_{S}^{2}}{\sigma_{X}^{2}}}} \sqrt{\left(C_{X}^{2}+W(1-T) \frac{\sigma_{S}^{2}}{\mu_{X}^{2}}\right)} C_{y}, \\
\operatorname{MSE}\left(\hat{\mu}_{P P}\right) \cong\left(\frac{1-f}{n}\right) \mu_{X}^{2}\left(C_{X}^{2}+W(1-T) \frac{\sigma_{S}^{2}}{\mu_{X}^{2}}+C_{Y}^{2}+2 \frac{\rho_{X Y}}{\sqrt{\left(1+W(1-T) \frac{\sigma_{S}^{2}}{\sigma_{X}^{2}}\right.}} \sqrt{\left(C_{X}^{2}+W(1-T) \frac{\sigma_{S}^{2}}{\mu_{X}^{2}}\right)} C_{Y}\right) .
\end{gathered}
$$




\subsection{Regression Estimator}

The proposed regression estimator is given by

$$
\hat{\mu}_{\text {Re } g P}=\bar{z}^{*}+\hat{\beta}_{Z^{*} Y}\left(\mu_{Y}-\bar{y}\right),
$$

Where $\hat{\beta}_{Z^{*} Y}$ is the sample regression coefficient between $Z^{*}$ and $Y$. The bias and MSE of the proposed regression estimator $\hat{\mu}_{\mathrm{Re} g P}$ is given by

$$
\operatorname{Bias}\left(\hat{\mu}_{\operatorname{Re} g P}\right) \cong-\beta_{Z^{*} Y}\left(\frac{1-f}{n}\right)\left\{\frac{\mu_{12}}{\mu_{11}}-\frac{\mu_{03}}{\mu_{02}}\right\},
$$

Where $\beta_{Z^{*} Y}=\beta_{X Y}$ and $\mu_{r s}=\frac{1}{N-1} \sum_{i=1}^{N}\left(Z_{i}^{*}-\bar{Z}^{*}\right)^{r}\left(y_{i}-\bar{Y}\right)^{s}$

$$
\operatorname{MSE}\left(\hat{\mu}_{\mathrm{Re} g P}\right) \cong\left(\frac{1-f}{n}\right) \mu_{z^{*}}^{2} C_{z^{*}}^{2}\left\{1-\rho_{Y Z^{*}}^{2}\right\},
$$

Now, replacing the value of $C_{Z^{*}}^{2}$ and $\rho_{Y Z^{*}}$ in above expressions, we get following expressions of bias and MSE of $\hat{\mu}_{\mathrm{Re} g P}$.

$$
\begin{aligned}
& \operatorname{Bias}\left(\hat{\mu}_{\mathrm{Re} g P}\right) \cong-\beta_{X Y}\left(\frac{1-f}{n}\right)\left\{\frac{\mu_{12}}{\mu_{11}}-\frac{\mu_{03}}{\mu_{02}}\right\}, \\
& \operatorname{MSE}\left(\hat{\mu}_{\mathrm{Re} g P}\right) \cong\left(\frac{1-f}{n}\right) \mu_{X}^{2}\left(C_{X}^{2}+W(1-T) \frac{\sigma_{S}^{2}}{\mu_{X}^{2}}\right)\left\{1-\frac{\rho_{X Y}^{2}}{1+W(1-T) \frac{\sigma_{S}^{2}}{\sigma_{X}^{2}}}\right\}, \\
& \operatorname{MSE}\left(\hat{\mu}_{\mathrm{Re} g P}\right) \cong\left(\frac{1-f}{n}\right) \sigma_{X}^{2}\left\{\left(1+W(1-T) \frac{\sigma_{S}^{2}}{\sigma_{X}^{2}}\right)-\rho_{X Y}^{2}\right\} .
\end{aligned}
$$

Again, note that for $W=1$ and $T=0$, the MSE of the proposed regression estimator coincides with the MSE of Gupta et al. (2012) proposed regression estimator and for $T=0$, the MSE of our proposed regression estimator is same as the MSE of Gupta et al. (2014) proposed estimator. 


\section{Algebraic and Numerical Comparisons}

In this section, we compare proposed ratio, product and regression estimators with Sousa et al. (2010), Gupta et al. (2012) and Gupta et al. (2014) estimators both algebraically and numerically, and establish the superiority of our proposed ratio, product and regression estimators. For comparing different estimators, we consider the Percent Relative Efficiency (PRE) as the performance criterion.

\subsection{Relative efficiency comparisons of different Ratio Estimators}

(i) The proposed ratio estimator will be more efficient than the Sousa et al. (2010) ratio estimator if

$$
\begin{aligned}
& \operatorname{MSE}\left(\hat{\mu}_{R P}\right)<\operatorname{MSE}\left(\hat{\mu}_{R S}\right) \\
& (1-W(1-T)) \frac{\sigma_{s}^{2}}{\mu_{x}^{2}}-2 \rho_{X Y} C_{Y}\left(\frac{\sqrt{\left(C_{X}^{2}+\frac{\sigma_{s}^{2}}{\mu_{x}^{2}}\right)}}{\sqrt{\left(1+\frac{\sigma_{s}^{2}}{\sigma_{x}^{2}}\right)}}-\frac{\sqrt{\left(C_{X}^{2}+W(1-T) \frac{\sigma_{s}^{2}}{\mu_{x}^{2}}\right)}}{\sqrt{\left(1+W(1-T) \frac{\sigma_{s}^{2}}{\sigma_{x}^{2}}\right)}}\right)>0 \\
& (1-W(1-T)) \frac{\sigma_{s}^{2}}{\mu_{x}^{2}} \sqrt{\left(1+\frac{\sigma_{s}^{2}}{\sigma_{x}^{2}}\right)\left(1+W(1-T) \frac{\sigma_{s}^{2}}{\sigma_{x}^{2}}\right)}-2 \rho_{X Y} C_{Y}\left(\sqrt{\left(C_{X}^{2}+\frac{\sigma_{s}^{2}}{\mu_{x}^{2}}\right)\left(1+W(1-T) \frac{\sigma_{s}^{2}}{\sigma_{x}^{2}}\right)}-\sqrt{\left(C_{X}^{2}+W(1-T) \frac{\sigma_{s}^{2}}{\mu_{x}^{2}}\right)\left(1+\frac{\sigma_{s}^{2}}{\sigma_{x}^{2}}\right)}>0\right.
\end{aligned}
$$

Now, consider only the second term

$$
\begin{aligned}
& \sqrt{\left(C_{X}^{2}+\frac{\sigma_{s}^{2}}{\mu_{x}^{2}}\right)\left(1+W(1-T) \frac{\sigma_{s}^{2}}{\sigma_{x}^{2}}\right)}-\sqrt{\left(C_{X}^{2}+W(1-T) \frac{\sigma_{s}^{2}}{\mu_{x}^{2}}\right)\left(1+\frac{\sigma_{s}^{2}}{\sigma_{x}^{2}}\right)} \\
= & \sqrt{\left(C_{X}^{2}+\frac{\sigma_{s}^{2}}{\mu_{x}^{2}}+W(1-T)\left(C_{X}^{2}+\frac{\sigma_{s}^{2}}{\mu_{x}^{2}} \frac{\sigma_{s}^{2}}{\sigma_{x}^{2}}\right)\right.}-\sqrt{\left(C_{X}^{2}+W(1-T) \frac{\sigma_{s}^{2}}{\mu_{x}^{2}}+\left(C_{X}^{2}+W(1-T) \frac{\sigma_{s}^{2}}{\mu_{x}^{2}}\right) \frac{\sigma_{s}^{2}}{\sigma_{x}^{2}}\right)} \\
= & \sqrt{\left(C_{X}^{2}+\frac{\sigma_{s}^{2}}{\mu_{x}^{2}}+W(1-T) C_{X}^{2} \frac{\sigma_{s}^{2}}{\sigma_{x}^{2}}+W(1-T) \frac{\left(\sigma_{s}^{2}\right)^{2}}{\mu_{x}^{2} \sigma_{x}^{2}}\right)}-\sqrt{\left(C_{X}^{2}+W(1-T) \frac{\sigma_{s}^{2}}{\mu_{x}^{2}}+C_{X}^{2} \frac{\sigma_{s}^{2}}{\sigma_{x}^{2}}+W(1-T) \frac{\left(\sigma_{s}^{2}\right)^{2}}{\mu_{x}^{2} \sigma_{x}^{2}}\right)} \\
= & 0
\end{aligned}
$$


Thus, the above inequality becomes

$$
\begin{aligned}
& (1-W(1-T)) \frac{\sigma_{s}^{2}}{\sigma_{x}^{2}} \sqrt{\left(1+\frac{\sigma_{s}^{2}}{\sigma_{x}^{2}}\right)\left(1+W(1-T) \frac{\sigma_{s}^{2}}{\sigma_{x}^{2}}\right)}-2 \rho_{X Y} C_{Y}(0)>0 \\
& (1-W(1-T)) \frac{\sigma_{s}^{2}}{\sigma_{x}^{2}} \sqrt{\left(1+\frac{\sigma_{s}^{2}}{\sigma_{x}^{2}}\right)\left(1+W(1-T) \frac{\sigma_{s}^{2}}{\sigma_{x}^{2}}\right)}>0
\end{aligned}
$$

The above expression is always true because all the quantities involved in the expression on right hand side are positive.

(ii) The proposed ratio estimator of the population mean performs better than Gupta et al. (2014) estimator if

$$
\operatorname{MSE}\left(\hat{\mu}_{R P}\right)<\operatorname{MSE}\left(\hat{\mu}_{R W G}\right)
$$

$W T \frac{\sigma_{s}^{2}}{\mu_{x}^{2}}-2 \rho_{X Y} C_{Y}\left(\frac{\sqrt{\left(C_{X}^{2}+W \frac{\sigma_{s}^{2}}{\mu_{x}^{2}}\right)}}{\sqrt{\left(1+W \frac{\sigma_{s}^{2}}{\sigma_{x}^{2}}\right)}}-\frac{\sqrt{\left(C_{X}^{2}+W(1-T) \frac{\sigma_{s}^{2}}{\mu_{x}^{2}}\right)}}{\sqrt{\left(1+W(1-T) \frac{\sigma_{s}^{2}}{\sigma_{x}^{2}}\right)}}\right)>0$

$$
W T \frac{\sigma_{S}^{2}}{\mu_{X}^{2}} \sqrt{\left(1+W \frac{\sigma_{S}^{2}}{\sigma_{X}^{2}}\right)\left(1+W(1-T) \frac{\sigma_{S}^{2}}{\sigma_{X}^{2}}\right)}-2 \rho_{X Y} C_{Y}\left(\sqrt{\left(C_{X}^{2}+W \frac{\sigma_{s}^{2}}{\mu_{x}^{2}}\right)\left(1+W(1-T) \frac{\sigma_{S}^{2}}{\sigma_{X}^{2}}\right)}-\sqrt{\left(C_{X}^{2}+W(1-T) \frac{\sigma_{S}^{2}}{\mu_{X}^{2}}\right)\left(1+W \frac{\sigma_{S}^{2}}{\sigma_{X}^{2}}\right)}\right)>0
$$

Now, consider the second term only

$$
\begin{aligned}
& \sqrt{\left(C_{X}^{2}+W \frac{\sigma_{s}^{2}}{\mu_{x}^{2}}\right)\left(1+W(1-T) \frac{\sigma_{S}^{2}}{\sigma_{X}^{2}}\right)}-\sqrt{\left(C_{X}^{2}+W(1-T) \frac{\sigma_{S}^{2}}{\mu_{X}^{2}}\right)\left(1+W \frac{\sigma_{S}^{2}}{\sigma_{X}^{2}}\right)} \\
& =\sqrt{\left(C_{X}^{2}+W \frac{\sigma_{s}^{2}}{\mu_{x}^{2}}+W(1-T)\left(C_{X}^{2}+W \frac{\sigma_{s}^{2}}{\mu_{x}^{2}}\right) \frac{\sigma_{S}^{2}}{\sigma_{X}^{2}}\right)}-\sqrt{\left(C_{X}^{2}+W(1-T) \frac{\sigma_{S}^{2}}{\mu_{X}^{2}}+W\left(C_{X}^{2}+W(1-T) \frac{\sigma_{S}^{2}}{\mu_{X}^{2}}\right) \frac{\sigma_{S}^{2}}{\sigma_{X}^{2}}\right)}
\end{aligned}
$$




$$
\begin{aligned}
& =\sqrt{\left(C_{X}^{2}+W \frac{\sigma_{s}^{2}}{\mu_{x}^{2}}+W(1-T) C_{X}^{2} \frac{\sigma_{S}^{2}}{\sigma_{X}^{2}}+W^{2}(1-T) \frac{\left(\sigma_{S}^{2}\right)^{2}}{\mu_{x}^{2} \sigma_{X}^{2}}\right)}-\sqrt{\left(C_{X}^{2}+W(1-T) \frac{\sigma_{S}^{2}}{\mu_{X}^{2}}+W C_{X}^{2} \frac{\sigma_{S}^{2}}{\sigma_{X}^{2}}+W^{2}(1-T) \frac{\left(\sigma_{S}^{2}\right)^{2}}{\mu_{X}^{2} \sigma_{X}^{2}}\right.} \\
& =0
\end{aligned}
$$

The above inequality becomes

$$
\begin{aligned}
& W T \frac{\sigma_{S}^{2}}{\mu_{X}^{2}} \sqrt{\left(1+W \frac{\sigma_{S}^{2}}{\sigma_{X}^{2}}\right)\left(1+W(1-T) \frac{\sigma_{S}^{2}}{\sigma_{X}^{2}}\right)}-2 \rho_{X Y} C_{Y}(0)>0 \\
& W T \frac{\sigma_{S}^{2}}{\mu_{X}^{2}} \sqrt{\left(1+W \frac{\sigma_{S}^{2}}{\sigma_{X}^{2}}\right)\left(1+W(1-T) \frac{\sigma_{S}^{2}}{\sigma_{X}^{2}}\right)}>0
\end{aligned}
$$

The above expression is always true because all the terms involved on right hand side are positive.

\subsection{Relative efficiency comparison of different Product estimators}

(i)The proposed product estimator performed better than Gupta et al. (2014) suggested product estimator if

$$
\operatorname{MSE}\left(\hat{\mu}_{P P}\right)<\operatorname{MSE}\left(\hat{\mu}_{P W G}\right)
$$

$W T \frac{\sigma_{s}^{2}}{\mu_{x}^{2}}+2 \rho_{X Y} C_{Y}\left(\frac{\sqrt{\left(C_{X}^{2}+W \frac{\sigma_{s}^{2}}{\mu_{x}^{2}}\right)}}{\sqrt{\left(1+W \frac{\sigma_{s}^{2}}{\sigma_{x}^{2}}\right)}}-\frac{\sqrt{\left(C_{X}^{2}+W(1-T) \frac{\sigma_{s}^{2}}{\mu_{x}^{2}}\right)}}{\sqrt{\left(1+W(1-T) \frac{\sigma_{s}^{2}}{\sigma_{x}^{2}}\right)}}>0\right.$

$$
W T \frac{\sigma_{S}^{2}}{\mu_{X}^{2}} \sqrt{\left(1+W \frac{\sigma_{S}^{2}}{\sigma_{X}^{2}}\right)\left(1+W(1-T) \frac{\sigma_{S}^{2}}{\sigma_{X}^{2}}\right)}+2 \rho_{X Y} C_{Y}\left(\sqrt{\left(C_{X}^{2}+W \frac{\sigma_{S}^{2}}{\mu_{x}^{2}}\right)\left(1+W(1-T) \frac{\sigma_{S}^{2}}{\sigma_{X}^{2}}\right)}-\sqrt{\left(C_{X}^{2}+W(1-T) \frac{\sigma_{S}^{2}}{\mu_{X}^{2}}\right)\left(1+W \frac{\sigma_{S}^{2}}{\sigma_{X}^{2}}\right)}\right)>0
$$

As we have already proved that 
$\sqrt{\left(C_{X}^{2}+W \frac{\sigma_{s}^{2}}{\mu_{x}^{2}}\right)\left(1+W(1-T) \frac{\sigma_{S}^{2}}{\sigma_{X}^{2}}\right)}-\sqrt{\left(C_{X}^{2}+W(1-T) \frac{\sigma_{S}^{2}}{\mu_{X}^{2}}\right)\left(1+W \frac{\sigma_{S}^{2}}{\sigma_{X}^{2}}\right)}=0$,

we have

$$
\begin{aligned}
& W T \frac{\sigma_{S}^{2}}{\mu_{X}^{2}} \sqrt{\left(1+W \frac{\sigma_{S}^{2}}{\sigma_{X}^{2}}\right)\left(1+W(1-T) \frac{\sigma_{S}^{2}}{\sigma_{X}^{2}}\right)}-2 \rho_{X Y} C_{Y}(0)>0 \\
& W T \frac{\sigma_{S}^{2}}{\mu_{X}^{2}} \sqrt{\left(1+W \frac{\sigma_{S}^{2}}{\sigma_{X}^{2}}\right)\left(1+W(1-T) \frac{\sigma_{S}^{2}}{\sigma_{X}^{2}}\right)}>0 .
\end{aligned}
$$

The above expression is always true because all the involved terms are positive.

\subsection{Relative efficiency comparison of different Regression estimators}

(i) The proposed regression estimator performs better than usual regression estimators if

$$
\begin{aligned}
& \operatorname{MSE}\left(\hat{\mu}_{\mathrm{Re} g P}\right)<\operatorname{MSE}\left(\hat{\mu}_{X P}\right) \\
& \left(\frac{1-f}{n}\right) \sigma_{X}^{2}\left(1+W(1-T) \frac{\sigma_{S}^{2}}{\sigma_{X}^{2}}-\rho_{X Y}^{2}\right)<\left(\frac{1-f}{n}\right) \sigma_{X}^{2}\left(1+W(1-T) \frac{\sigma_{S}^{2}}{\sigma_{X}^{2}}\right) \\
& \rho_{X Y}^{2}>0
\end{aligned}
$$

Now we observe from above expressions, $\operatorname{MSE}\left(\hat{\mu}_{\operatorname{Reg} P}\right)$ is always less than $\operatorname{MSE}\left(\hat{\mu}_{X P}\right)$ because $\rho_{X Y}^{2}>0$, which is always true.

(ii)The proposed regression estimator performs better than Gupta et al. (2012) regression estimator if

$$
\begin{aligned}
& \operatorname{MSE}\left(\hat{\mu}_{\mathrm{Re} g P}\right)<\operatorname{MSE}\left(\hat{\mu}_{\mathrm{Re} g G}\right) \\
& \left(\frac{1-f}{n}\right) \sigma_{X}^{2}\left(1+W(1-T) \frac{\sigma_{S}^{2}}{\sigma_{X}^{2}}-\rho_{X Y}^{2}\right)<\left(\frac{1-f}{n}\right) \sigma_{X}^{2}\left(1+\frac{\sigma_{S}^{2}}{\sigma_{X}^{2}}-\rho_{X Y}^{2}\right)
\end{aligned}
$$


$1+W(1-T) \frac{\sigma_{S}^{2}}{\sigma_{X}^{2}}-\rho_{X Y}^{2}<1+\frac{\sigma_{S}^{2}}{\sigma_{X}^{2}}-\rho_{X Y}^{2}$

$W(1-T) \frac{\sigma_{S}^{2}}{\sigma_{X}^{2}}<\frac{\sigma_{S}^{2}}{\sigma_{X}^{2}}$

$W(1-T)<1$.

The above expression $W(1-T)<1$ is always true because both $T$ and $W$ lie between 0 and $1(0<T<1,0 \leq W<1)$.

(iii) The proposed regression estimator performs better than Gupta et al. (2014) regression estimator if

$$
\begin{aligned}
& \operatorname{MSE}\left(\hat{\mu}_{\mathrm{Re} g P}\right)<\operatorname{MSE}\left(\hat{\mu}_{\mathrm{Re} g W G}\right) \\
& \left(\frac{1-f}{n}\right) \sigma_{X}^{2}\left(1+W(1-T) \frac{\sigma_{S}^{2}}{\sigma_{X}^{2}}-\rho_{X Y}^{2}\right)<\left(\frac{1-f}{n}\right) \sigma_{X}^{2}\left(1+W \frac{\sigma_{S}^{2}}{\sigma_{X}^{2}}-\rho_{X Y}^{2}\right) \\
& 1+W(1-T) \frac{\sigma_{S}^{2}}{\sigma_{X}^{2}}-\rho_{X Y}^{2}<1+W \frac{\sigma_{S}^{2}}{\sigma_{X}^{2}}-\rho_{X Y}^{2} \\
& W(1-T) \frac{\sigma_{S}^{2}}{\sigma_{X}^{2}}<W \frac{\sigma_{S}^{2}}{\sigma_{X}^{2}} \\
& 1-T<1 \\
& T>0
\end{aligned}
$$

Which is always true because $T$ always lies between 0 and 1

\subsection{Numerical Comparisons}

To know the extent of the $P R E$ of different proposed estimator, we calculated the $P R E$ numerically. The values of the parameters are fixed same as considered by Gupta et al. (2014). The PREs of the different estimators are defined as

$$
P R E=\frac{M S E\left(\hat{\mu}_{X S}\right)}{M S E\left(\hat{\mu}_{i}\right)} \times 100
$$


Where $i=X S, R S, \operatorname{Re} g G, R W G, \operatorname{Re} g W G, X P, R P, \operatorname{Re} g P$.

We considered $\quad N=5000, n=200, \rho_{X Y}=0.8, \mu_{X}=6, \mu_{Y}=4, \sigma_{X}=3$, $\sigma_{S}=3, \quad \sigma_{Y}=2, W=0.1,0.2, \ldots, 1.0 \quad$ and $T=0.1,0.3,0.5,0.7,0.9 \quad$ and calculated PRE of each estimator as defined above. The PRE results, so obtained, are presented in the Table 1, below.

Table 1: PRE comparison of different estimators when $N=5000, n=200$, $\rho_{X Y}=0.8, \mu_{X}=6, \mu_{Y}=4, \sigma_{X}=3, \sigma_{S}=3$

\begin{tabular}{|c|c|c|c|c|c|c|c|c|}
\hline & $\hat{\mu}_{X s}$ & $\hat{\mu}_{R S}$ & $\hat{\mu}_{\text {RegG }}$ & $\hat{\mu}_{\text {RWG }}$ & $\hat{\mu}_{\text {RegWG }}$ & $\hat{\mu}_{X P}$ & $\hat{\mu}_{R P}$ & $\hat{\mu}_{\text {RegP }}$ \\
\hline$W$ & \multicolumn{8}{|c|}{$T=0.1$} \\
\hline 0.1 & 100 & 142.85 & 147.06 & 400 & 434.78 & 183.48 & 408.16 & 444.44 \\
\hline 0.2 & 100 & 142.85 & 147.06 & 333.33 & 357.14 & 169.49 & 344.82 & 370.37 \\
\hline 0.3 & 100 & 142.85 & 147.06 & 285.71 & 303.03 & 157.48 & 298.50 & 317.46 \\
\hline 0.4 & 100 & 142.85 & 147.06 & 250 & 263.15 & 147.05 & 263.15 & 277.77 \\
\hline 0.5 & 100 & 142.85 & 147.06 & 222.22 & 232.55 & 137.93 & 235.29 & 246.91 \\
\hline 0.6 & 100 & 142.85 & 147.06 & 200 & 208.33 & 129.87 & 212.76 & 222.22 \\
\hline 0.7 & 100 & 142.85 & 147.06 & 181.81 & 188.67 & 122.69 & 194.17 & 202.02 \\
\hline 0.8 & 100 & 142.85 & 147.06 & 166.66 & 172.41 & 116.27 & 178.57 & 185.18 \\
\hline 0.9 & 100 & 142.85 & 147.06 & 153.84 & 158.73 & 110.49 & 165.28 & 170.94 \\
\hline \multirow[t]{2}{*}{1} & 100 & 142.85 & 147.06 & 142.85 & 147.05 & 105.26 & 153.84 & 158.73 \\
\hline & \multicolumn{8}{|c|}{$T=0.3$} \\
\hline 0.1 & 100 & 142.85 & 147.06 & 400 & 434.78 & 186.91 & 425.53 & 465.11 \\
\hline 0.2 & 100 & 142.85 & 147.06 & 333.33 & 357.14 & 175.44 & 370.37 & 400 \\
\hline 0.3 & 100 & 142.85 & 147.06 & 285.71 & 303.03 & 165.29 & 327.87 & 350.88 \\
\hline 0.4 & 100 & 142.85 & 147.06 & 250 & 263.15 & 156.25 & 294.12 & 312.5 \\
\hline 0.5 & 100 & 142.85 & 147.06 & 222.22 & 232.55 & 148.15 & 266.67 & 281.69 \\
\hline 0.6 & 100 & 142.85 & 147.06 & 200 & 208.33 & 140.85 & 243.90 & 256.41 \\
\hline 0.7 & 100 & 142.85 & 147.06 & 181.81 & 188.67 & 134.23 & 224.72 & 235.29 \\
\hline 0.8 & 100 & 142.85 & 147.06 & 166.66 & 172.41 & 128.21 & 208.33 & 217.39 \\
\hline 0.9 & 100 & 142.85 & 147.06 & 153.84 & 158.73 & 122.69 & 194.17 & 202.02 \\
\hline \multirow[t]{2}{*}{1} & 100 & 142.85 & 147.06 & 142.85 & 147.05 & 117.64 & 153.84 & 158.73 \\
\hline & \multicolumn{8}{|c|}{$T=0.5$} \\
\hline 0.1 & 100 & 142.85 & 147.06 & 400 & 434.78 & 190.48 & 444.44 & 487.81 \\
\hline 0.2 & 100 & 142.85 & 147.06 & 333.33 & 357.14 & 181.82 & 400 & 434.78 \\
\hline 0.3 & 100 & 142.85 & 147.06 & 285.71 & 303.03 & 173.91 & 363.64 & 392.16 \\
\hline 0.4 & 100 & 142.85 & 147.06 & 250 & 263.15 & 166.67 & 333.3 & 357.14 \\
\hline
\end{tabular}




\begin{tabular}{|r|c|c|c|c|c|c|c|c|}
\hline 0.5 & 100 & 142.85 & 147.06 & 222.22 & 232.55 & 160 & 307.69 & 327.87 \\
\hline 0.6 & 100 & 142.85 & 147.06 & 200 & 208.33 & 153.85 & 285.71 & 303.03 \\
\hline 0.7 & 100 & 142.85 & 147.06 & 181.81 & 188.67 & 148.15 & 266.67 & 281.69 \\
\hline 0.8 & 100 & 142.85 & 147.06 & 166.66 & 172.41 & 142.86 & 250 & 263.16 \\
\hline 0.9 & 100 & 142.85 & 147.06 & 153.84 & 158.73 & 137.93 & 235.29 & 246.91 \\
\hline 1 & 100 & 142.85 & 147.06 & 142.85 & 147.05 & 133.33 & 222.22 & 232.56 \\
\hline \multicolumn{8}{|c|}{$T=0.7$} \\
\hline 0.1 & 100 & 142.85 & 147.06 & 400 & 434.78 & 194.17 & 465.12 & 512.82 \\
\hline 0.2 & 100 & 142.85 & 147.06 & 333.33 & 357.14 & 188.68 & 434.78 & 476.19 \\
\hline 0.3 & 100 & 142.85 & 147.06 & 285.71 & 303.03 & 183.49 & 408.16 & 444.44 \\
\hline 0.4 & 100 & 142.85 & 147.06 & 250 & 263.15 & 178.57 & 384.62 & 416.67 \\
\hline 0.5 & 100 & 142.85 & 147.06 & 222.22 & 232.55 & 173.91 & 363.64 & 392.17 \\
\hline 0.6 & 100 & 142.85 & 147.06 & 200 & 208.33 & 169.49 & 344.83 & 370.37 \\
\hline 0.7 & 100 & 142.85 & 147.06 & 181.81 & 188.67 & 165.29 & 327.87 & 350.88 \\
\hline 0.8 & 100 & 142.85 & 147.06 & 166.66 & 172.41 & 161.29 & 312.5 & 333.33 \\
\hline 0.9 & 100 & 142.85 & 147.06 & 153.84 & 158.73 & 157.48 & 298.51 & 317.46 \\
\hline 1 & 100 & 142.85 & 147.06 & 142.85 & 147.05 & 153.84 & 285.71 & 303.03 \\
\hline \multicolumn{7}{|c|}{$T$} \\
\hline 0.1 & 100 & 142.85 & 147.06 & 400 & 434.78 & 198.02 & 487.80 & 540.54 \\
\hline 0.2 & 100 & 142.85 & 147.06 & 333.33 & 357.14 & 196.08 & 476.19 & 526.32 \\
\hline 0.3 & 100 & 142.85 & 147.06 & 285.71 & 303.03 & 194.17 & 465.12 & 512.82 \\
\hline 0.4 & 100 & 142.85 & 147.06 & 250 & 263.15 & 192.31 & 454.55 & 500 \\
\hline 0.5 & 100 & 142.85 & 147.06 & 222.22 & 232.55 & 190.48 & 444.44 & 487.80 \\
\hline 0.6 & 100 & 142.85 & 147.06 & 200 & 208.33 & 188.68 & 434.78 & 476.19 \\
\hline 0.7 & 100 & 142.85 & 147.06 & 181.81 & 188.67 & 186.92 & 425.53 & 465.11 \\
\hline 0.8 & 100 & 142.85 & 147.06 & 166.66 & 172.41 & 185.19 & 416.67 & 454.54 \\
\hline 0.9 & 100 & 142.85 & 147.06 & 153.84 & 158.73 & 183.48 & 408.16 & 444.44 \\
\hline 1 & 100 & 142.85 & 147.06 & 142.85 & 147.05 & 181.81 & 400 & 434.78 \\
\hline
\end{tabular}

From Table 1, it is observed that $P R E$ of the proposed ratio estimator is greater than 100 for all values of $T(0<T<1)$ which proves the superiority of our proposed estimator over usual mean per unit estimator of Sousa et al. (2010). The PRE of the proposed ratio estimator is greater than $P R E$ of Sousa et al. (2010) ratio estimator and Gupta et al. (2014) ratio estimator for all values of $T(0<T<1)$ and $\rho_{X Y}^{2}>0$. From Tables 1-5, it can also be observed that as the value of $T$ increases the PRE of the proposed ratio estimator increases. The $P R E$ of the proposed regression estimator is greater than 100 for all values of $T$ and $\rho_{X Y}^{2}>0$. Thus, the proposed 
Regression estimator proves to be superior than Sousa et al. (2010). The PRE of the proposed regression estimator is greater than Gupta et al. (2012) regression estimator and Gupta et al. (2014) regression estimator for all values of $T(0<T<1)$ and $\rho_{X Y}^{2}>0$. As expected, when the value of $T$ increases, the efficiency of our proposed regression estimator also increases.

\section{Conclusion}

The use of auxiliary information is proved to be very useful in enhancing the efficiency of the estimators. Sousa et al. (2010) used a regression type estimator of finite population mean which is based on an additive scrambled response model. Gupta et al. (2012) proposed a regression type estimator which showed a significant improvement in the efficiency of the estimator. Gupta et al. (2014) proposed an optional RRT. They used a ratio and regression estimators for improving efficiency of the estimates and showed the superiority of their results over Sousa et al. (2010) and Gupta et al. (2012). We used a two-stage ORR model and proposed ratio, product and regression type estimator utilizing non-sensitive auxiliary information. Our proposed ratio, product and regression estimators perform better than Sousa et al. (2010), Gupta et al. (2012) and Gupta et al. (2014) estimators.

\section{References}

1. Abel, G. J. Barakat, B. Samir K. C. and Lutz, W., (2016). Meeting the sustainable development

2. Goals leads to lower world population growth. National Academy of Sciences of the United States of America (PNAS). 113 (50), 14294-14299.

3. Bar-Lev, S. K. E., Bobovitch, E. \& Boukai, B., (2004). A note on randomized response models for quantitative data. Metrika. (60), 225-250.

4. Blume, A., Board, O. J and Kawamura, K., (2007). Noisy Talk. Theoretical Economics. (2), 395-440.

5. Bhargava, M., Singh, R. (2000). A Modified Randomization Device for Warner's Model. Statistica, 60:315-321.

6. Chaudhuri, A., Mukerjee, R. (1985). Optionally Randomized Response Techniques. Calcutta Statistical Association Bulletin, 34:225-229.

7. Cronin Jr, et al. (2000). Assessing the effects of quality, value, and customer satisfaction on consumer behavioral intentions in service environments. Journal of Retailing. 76(2), 193-218.

8. Cruyff, M. F., Ulf Böckenholt Peter G. M. and van der Heijden, (2016). The multidimensional randomized response design: Estimating different aspects of the same sensitive behavior. Behavior Research Methods. (48), 390-399. 
9. Diana, G., Perri, P.F. (2007). Estimation of Finite Population Mean Using Multi-Auxiliary Information. Metron, LXV 99-112.

10. Dawidowska, M., Kraszewska, M. D., Derwich, K. and Szczepenski, T. (2012), In Molecular Aspects of Hematologic Malignancies: Diagnostic Tools and Clinical Application. (Witt. M., Dawidowska, M. and Szczepenski, T., eds), Springer: Berlin, 3-28.

11. Eichhorn, B.H. and Hayre, L.S., (1983). Scrambled randomized response methods for obtaining Sensitive quantitative dada. Journal of Statistics and planning Inference. (7), 307-316.

12. Gjestvang, C. R., Singh, S. (2007).Forced Quantitative Randomized Response Model: A New Device. Metrika, 66:243-257.

13. Greenberg, B. G., Abul-Ela Abdel-Latif, A., Simmons, W. R., Horvitz, D. G. (1969). The Unrelated Question RR Model: Theoretical Framework. Journal of the American Statistical Association, 64, 52-539.

14. Gupta, S. N., Gupta, B. C., Singh, S. (2002). Estimation of Sensitivity Level of Personal Interview Survey Questions. Journal of Statistical Planning and Inference, 100:239-247.

15. Gupta, S., Kalucha, G., Shabbir,J., Dass, B. K.(2014). Estimation of Finite Population Mean Using Optional RRT Models in the Presence of Nonsensitive Auxiliary Information. American Journal of Mathematical and Management Sciences, 31:1-13.

16. Gupta, S., Shabbir, J. and Sehra, S. (2010). Mean and Sensitivity Estimation in Optional Randomized Response Models. Journal of Statistical Planning and Inference 140(10): 2870-2874.4

17. Gupta, S., Shabbir, J., Sousa, R., Real, P. C.(2012). Estimation of the Mean of a Sensitive Variable in the Presence of Auxiliary Information, Communications in Statistics - Theory and Methods, 41:13-14, 2394-2404.

18. Gupta, S.N., Thornton, B., Shabbir, J., Singhal, S. (2006). A Comparison of Multiplicative and Additive Optional RRT Models. Journal of Statistical Theory and Applications, 5:226-239.

19. Groves, R., M., Floyd, J. Fowler, Jr., Mick, P., Couper, J. M., Lepkowski, E. S. \& Tourangeau, R. (2009). Survey Methodology. Hoboken, New Jersey: John Wiley and Sons, Inc.

20. Gupta, S., Shabir, J., Sousa, R. and Corte-Real, P. (2012). Estimation of the mean of a sensitive variable in the presence of auxiliary information. Communications in Statistics-Theory and Methods. 41, 23942404. 
21. Huang, K. C. (2008). Estimation for the Sensitive Characteristic Using Optional Randomized Response Technique. Quality and Quantity, 42, 679686.

22. Huang, K.C. (2010). Unbiased estimators of mean, variance and sensitivity level for quantitative characteristics in finite population sampling. Metrika. 71, 341-352.

23. Himmelfarb, S., Edgell, S.E. (1980). Additive Constant Model: A Randomized Response Technique for Eliminating Evasiveness to Quantitative Response Questions. Psychological Bulletin, 87:525-530.

24. Horvitz, D.G., Shah, B.V., Simmons, W.R. (1967). The Unrelated Question Randomized Response Model. Proc. Of Social Statistics Section, Amer. Statist. Assoc., 65-72.

25. Karelaia, N. and Hogarth, R. M. (2008). Determinants of linear judgment: A meta-analysis of lens studies. Psychological Bulletin. 134(3), 404-426.

26. Kelly, C. A., Soler-Hampejsek, E., Mensch, B. S. and Hewett, P. C. (2013). Social desirability bias in sexual behavior reporting: Evidence from an interview mode experiment in rural Malawi. International Perspectives on the Sexual and Reproductive Health. 1, 14-21.

27. Kellogg S. S., Rosenbaum, T. Y., Dweck, A., Millheiser, L., PillaiFriedman, S. and Krychman, M. (2014). Sexual health and religion: a primer for the sexual health clinician. Journal of Sexual Medicine. (7), 1607-1618.

28. Krumpal, I., (2012). Estimating the prevalence of xenophobia and antisemitism in Germany: a comparison of randomized response and direct questioning. Social Science Research. (41), 1387-1403.

29. Kuokkanen, H. (2017). Fictitious consumer responsibility? Quantifying social desirability bias in corporate social responsibility surveys. Palgrave Communications.3,16106,DOI:

10.1057/palcomms.2016.106,www.palgrave-journals.com/palcomms.

30. Kadilar, C., Cingi, H. (2006). Improvement in Estimating the Population Mean in Simple Random Sampling. Applied Mathematics Letters, 19(1): 75-79.

31. Koyuncy, N., Kadilar, C. (2009). Efficient Estimators For The Population Mean. Hacettepe Journal of Mathematics and Statistics, 38(2), pp. 217-225.

32. Kuk, A. Y. C. (1990). Asking Sensitive Questions Directly. Biometrika, 77:436-438.

33. Lee, Z. and Sargeant, A. (2011). Dealing with social desirability bias: An application to charitable giving. European Journal of Marketing. 45(5), 703-719. 
34. Lensvelt-Mulders, G. J., Hox, J. J., Van der Heijden, P. G. \& Maas, C. J. (2005). Meta-analysis of randomized response research: Thirty five years of validation. Sociological Methods and Research. 33, 319-348.

35. Mahmood, M., Singh, S., Horn, S. (1998). On the Confidentiality Guaranteed Under Randomized Response Sampling: A Comparison with Several New Techniques. Biometrical Journal, 40:237-242.

36. Mangat, N. S. (1994). An Improved Randomized Response Strategy. Journal of Royal Statistical Society: Series B, 56: 93-95.

37. Mangat, N.S., Singh, R. (1990). An Alternative Randomized Response Procedure. Biometrika, 77:439-442.

38. Mahoney, J., and Rueschemeyer, D. (2003). Comparative historical analysis in the social sciences. Cambridge, UK: Cambridge University Press.

39. McNabb, D. E., (2015). Research Methods for Political Science: Quantitative and Qualitative Methods $\left(2^{\text {nd }} E d\right)$. Taylor \& Francis, N. Y.

40. Odumade, O. and Singh, S. (2010). An alternative to the Bar-lev, Bobovitch, and Boukai randomized response model. Sociological Methods \& Research. 39(2), 206-221.

41. Ojikutu, M. and Bolanle, I. (2018). Anti-fertility effect of aqueous seed extract of buchholzia coriacea. . In Society for Endocrinology BES 2018, volume 59. Bio Scientifica.

42. Ryu, J.B., Kim, J.M., Heo, T.Y., Park, C. G.(2006). On stratified randomized response sampling. Model Assisted Statistics and Applications, 1:31-36.

43. Robinson, M. M. and Irwin, D. E. (2019), Are there two visual short-term memory stores? A state-trace analysis. Journal of Mathematical Psychology. (90), 23-30.

44. Schill, D., and Kirk, R. (2017). Angry, Passionate, and Divided: Undecided voters and the 2016 presidential election. American Behavioral Scientist, 61(9), 1056-1076.

45. Shah, S. F., Cheema, S. A., Hussain, Z., and Shah, E. A. (2020a). Masking data: a solution to social desirability bias in paired comparison experiments. Communications in Statistics Simulation and Computation, (in press). DOI: 10.1080/03610918.2019.1710191.

46. Shah, S. F., Hussain, Z., Riaz, M., and Cheema, S. A. (2020b). ShewhartType charts for masked data: A strategy for handling the privacy issue. Mathematical Problems in Engineering. Article ID 5104753, Vol. 2020, 111. 
47. Stecklov, G., Weinreb, A. A., \& Sana, M. (2015). Family planning for strangers: an experiment on the validity of reported contraceptive use. PloS ONE. 10(8).

48. Shabbir, J., Gupta, S. (2010). Estimation of the Finite Population Mean in Two-Phase Sampling when Auxiliary Variables are Attribute. Hacettepe Journal of Mathematics and Statistics, 39(1):121-129.

49. Singh, S., Horn, S., Singh, R., Mangat, N.S. (2003). On the Use of Modified Randomization Device for Estimating the Prevalence of a Sensitive Attribute. Statistics in Transition, 6(4): 515-522.

50. Sousa, R., Shabbir, J., Real, P. C., Gupta, S. (2010). Ratio Estimation of the Mean of a Sensitive Variable in the Presence of Auxiliary Information. Journal of Statistical Theory and Practice,4(3):495-507.

51. Tracy, D., Mangat, N. (1996). Some Development in Randomized Response Sampling During the Last Decade-A Follow Up of Review by Chaudhuri and Mukerjee. Journal of Applied Statistical Sciences, 4:533544.

52. Turgut, Y., Cingi, H.(2008). New Generalized Estimators for the Population Variance Using Auxiliary Information. Hacettepe Journal of Mathematics and Statistics, 37(2): 177-184.

53. Van Der Heijden, P. G., Van Gils, G., Bouts, J. A. N., \& Hox, J. J. (2000). A comparison of randomized response, computer-assisted self-interview, and face-to-face direct questioning: Eliciting sensitive information in the context of welfare and unemployment benefit. Sociological Methods \& Research. 28(4), 505-537.

54. Warner, S. L. (1965). Randomized Response: A Survey Technique for Eliminating Evasive Answer Bias. Journal of American Statistical Association, 60:63-69.

55. Zaizai, Y. (2005-2006). Ratio Method of Estimation of Population Proportion Using Randomized Response Technique. Model Assisted Statistics and Applications, 1:125-130. 